\title{
The Voxel Onset Time as a Method for the Evaluation of Two Photon Lithography
}

\author{
Sascha Engelhardt ${ }^{* 1,{ }^{* 2}}$, Jenny Tempeler ${ }^{* 1}$, Arnold Gillner ${ }^{* 2}$ and Martin Wehner ${ }^{* 2}$ \\ ${ }^{* 1}$ Institute for Laser Technology, RWTH Aachen, Steinbachstraße 15, 52074 Aachen, Germany \\ ${ }^{* 2}$ Fraunhofer Institute for Laser Technology, Steinbachstraße 15, 52074 Aachen, Germany \\ E-mail: sascha,engelhardt@ilt.fraunhofer.de
}

\begin{abstract}
Two photon lithography allows the fabrication of arbitrary 3D structures with possible applications as micromechanical and microelectromechanical systems, photonic devices, 3D cell culture systems and scaffolds for tissue engineering. In order to achieve maximum resolution the process parameters have to be perfectly fitted to a given material. Normally, this interaction is studied by measuring the size of the generated volume pixels (voxel). In general, these analyses are time consuming, since they necessitate careful sample preparation and the use of a scanning electron microscope. In this paper, the threshold time for voxel formation, the voxel onset time (VOT), is presented as a parameter, which can give additional insight in the process of voxel formation. VOT is measured by a simple optical method, which can be implemented easily in already existing two photon lithography setups. Since the VOT method is considerably faster than voxel size analysis, it could be used in the future for faster screening of novel materials, while giving additional input of time dependencies of voxel growth.
\end{abstract}

DOI: $10.2961 /$ jlmn.2013.03.0008

Keywords: Two photon lithography, voxel, crosslinking, two photon polymerization

\section{Introducion}

Two photon lithography (TPL), or direct laser writing is a versatile tool to generate arbitrary $3 \mathrm{D}$ structures with subdiffraction limited resolution [1,2,3,4]. A tightly focused laser beam delivers high photon densities in a confined space and time. The resulting high photon densities trigger a nonlinear optical effect in a photosensitive material, resulting in confined chemical polymerization, crosslinking, or photoactivation. Applications range from photonics to biomedicine $[5,6,7,8,9,10,11,12]$. Two main challenges are encountered at opposite sides of the size scale. On the one side, smaller feature sizes are desired, in order to enhance the functionality of the generated object. In the last couple of years, different strategies, such as stimulated emission depletion (STED) lithography, have been pursuit to achieve sub-100 nm feature resolution $[13,14,15,16]$. On the other hand, macroscopic products with high resolution features are necessary to translate this promising fabrication technology from the laboratory into industry. Enhancing the sensitivity of the employed material and parallelization through multifocal technologies are the two main strategies to achieve this aim $[17,18,19,20]$. In order to address these two challenges, achievable resolution and fabrication speed, a deep understanding of the interaction of laser irradiation and photosensitive material system is necessary. This interaction has been described mostly by measuring the size of crosslinked structures under different process conditions [21,22,23,24]. Single volume pixels (voxels) represent a snapshot of the polymerization process at a given time. Thus, their length and diameter have been used to study polymerization efficiency, kinetics and overall de- pendency on process parameters. However, this type of voxel analysis does not directly allow studying the time dependency of single voxel formation and for a kinetic study massive amounts of voxels have to be prepared and analyzed. Yet, voxel growth kinetic has a profound effect on minimal achievable resolution, as well as on possible process speed.

In this study, a method is presented, which measures voxel formation in-situ. In the case of radical induced polymerization, voxel formation is a process based on local crosslinking of a prepolymer, initiated by a photosensitive molecule called photoinitiator. This crosslinking results in a local change in density and therefore refractive index. With ongoing voxel formation the size of the crosslinked volume increases, as well as the crosslinking density. Structures possessing a different refractive index than their surrounding lead to light scattering and refraction. Scattering strongly depends on particle size and refractive index and is therefore a feasible tool to characterize voxel growth in situ. In this study, the threshold time for voxel formation, the voxel onset time (VOT), is measured based on the above described strategy.

\section{Materials and Methods}

The experimental setup for TPL (Fig. 1) consists of a tunable Ti:Sapphire laser source, with a pulse duration of approximately $100 \mathrm{fs}$, a repetition rate of $80 \mathrm{MHz}$ and a maximum mean laser power output of approximately $3 \mathrm{~W}$. The laser beam passes a $\lambda / 2$ waveplate and a polarizing beam splitter for attenuation, a mechanical shutter and a telescope, before being coupled in a microscope objective (NA 


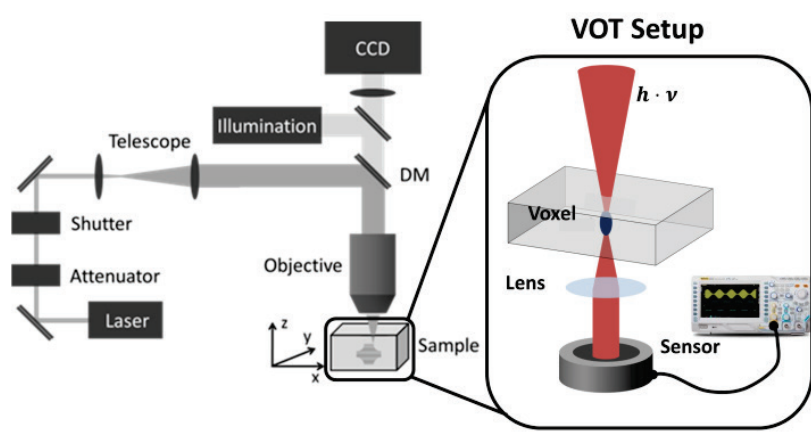

Fig. 1 Scheme of the experimental setup used for VOT measurement.

$0.6,40 \mathrm{X}$, air) by a dichroic mirror. The beam diameter is set to slightly overfill the back aperture of the objective in order to get an almost diffraction limited focal spot. Voxel growth can be monitored by a charge coupled device (CCD), situated behind the dichroic mirror and a tube lens. The illumination source is coupled for illumination by means of a beam splitter.

The photosensitive material is situated between a microscope slide and a cover slip, separated by glass spacers with a thickness of $170 \mu \mathrm{m}$. In this study three types of photosensitive material are used. The trifunctional monomer ethoxylated trimethylopropane triacrylate (SR499, Sartomer) is used as a monomer for radical polymerization. Two samples are prepared, adding either the photoinitiator Irgacure $^{\circledR} 184$ at a $\mathrm{w}-\%$ of $4 \%$, or the photoinitiator Irgacure $^{\circledR}$ OXE01 at a w- $\%$ of $0.3 \%$ (both BASF). The low concentration in the latter case is chosen due to the high sensitivity of the mixture, leading to rapid polymerization upon irradiation and even gelation under ambient light during sample preparation. As a third material system, a protein solution of $300 \mathrm{mg} / \mathrm{ml}$ bovine serum albumin (BSA) in double distilled water and $4 \mathrm{mmol}$ flavine mononucleotide (FMN) as photosensitizer is prepared (both SigmaAldrich).Voxels were generated in one of the three possible material systems at a time, by laser irradiation with a duration of $100 \mathrm{~ms}$.

The angle of the sample can be adjusted with respect to the incoming laser beam with the aid of a leveling platform. The transmitted laser irradiation is collected by a lens, situated below the material sample and passed on a InGaAs photodiode (DET10A/M, Thorlabs $\mathrm{GmbH}$ ). The signal is recorded with an oscilloscope (DPO7104 $1 \mathrm{GHz}$, Tektronix). The vertical position of the laser focus is adjusted by moving the microscope objective, while the horizontal translation is done by two translational stages. Horizontal movement encompasses the sample, the collection lens and the photodiode for VOT measurements.

\section{Results and Discussion}

Due to the above described optical setup the signal-tonoise ratio depends on the relative position of microscope objective (voxel formation) and collection lens (signal detection). Since the relative position of the microscope objective and the collection lens is changed upon sample movement, the horizontal and vertical distance is determined in which the transmission signal changes less than
$10 \%$ of the maximum value. For this reason, the transmission signal of several positions is recorded without a sample (Fig. 2). The horizontal sensitivity of the setup is high, since a movement of approximately $30 \mu \mathrm{m}$ suffices for a signal loss of $10 \%$ (Fig. 2 (a)). The signal dependency on the position follows a parabolic form. The signal dependency in vertical direction is linear over a range of approx. $300 \mu \mathrm{m}$ and less sensitive to the positional variation (Fig. 2 (b)). A signal reduction of $10 \%$ is associated with a motion of approx. $200 \mu \mathrm{m}$, i.e. over the whole thickness of the material sample. Here, the turning point of the measured signal is not shown, due to mechanical restraints. Due to the relative insensitivity of the signal on the focus depth, VOT measurements can be taken at different focusing depth.

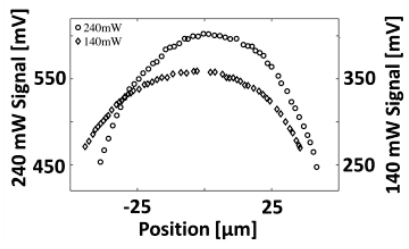

(a)

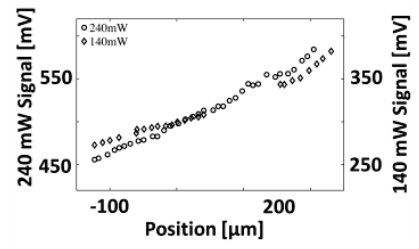

(b)
Fig. 2 Transmission signal variation for translation motion in horizontal (a) and vertical (b) direction.

In two photon based lithography, voxels are formed in the focal region of the incoming laser beam. Hence, almost the entire laser intensity $I_{0}$ is influenced by the refractive index change, associated with the crosslinking process of voxel formation (Fig. 3). The influences can be summarized to reflection, refraction, scattering and absorption. The measured transmitted laser beam contains in part each of these influences. Due to the low absorption coefficient of the material systems in the near infrared region, absorption effects can be neglected. The amount of reflection is also neglectable, as confirmed by VOT measurements in a conjugated back focal plane (data not shown). Thus, the transmitted signal is mostly contributed to a combination of scattering and refraction effects. Since voxel formation includes constant changes in density and size, it can be expected that the transmission signal has a complex and non-trivial form. In

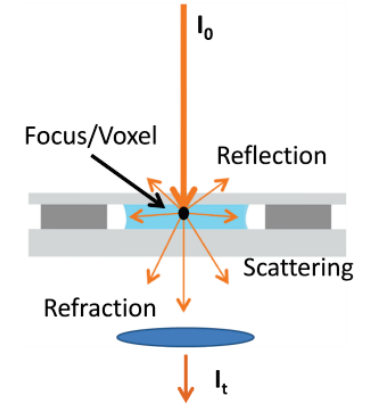

Fig. 3 The transmitted intensity is strongly influenced by refraction and scatterin effects. the case of radical polymerization with the photoinitiator Irgacure $^{\circledR} 184$, the VOT can be clearly detected as a sharp and sudden loss in transmission signal (Fig.4 (a)). Depending on the applied process parameters, typical VOT range from several milliseconds to $100 \mathrm{~ms}$. The signal after VOT shows drastic undulations and variation in magnitude. Most probably, these oscillations have their origin in a combination of Brownian motion of the voxels and changes in scattering and refraction characteristics caused by the constant change in voxel density and size. Additionally, the focused laser beam leads in part to optical trapping of the voxel. 


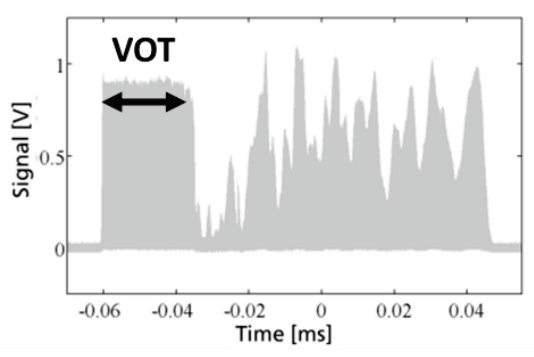

(a)

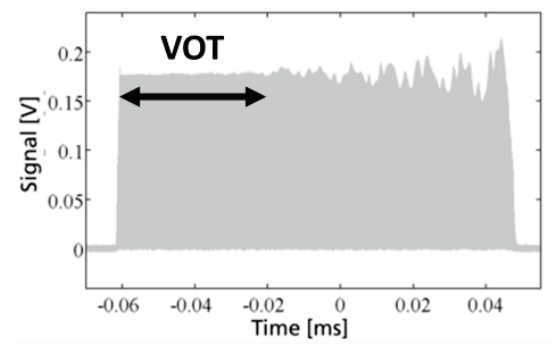

(b)

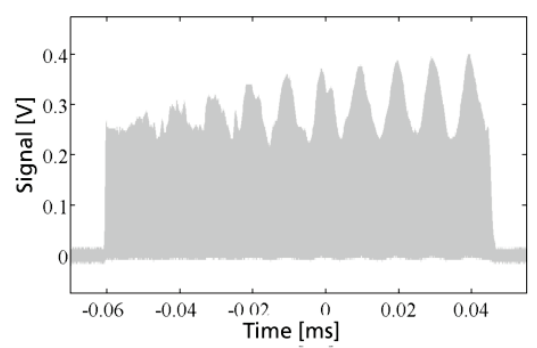

(c)

Fig. 4 Transmission signals for: (a) radical polymerization using Irgacure $^{\circledR} 184$, (b) protein solution and (c) radical polymerization using Irgacure ${ }^{\circledR}$ OXE01. VOT can be detected for (a) and (b), while for (c) polymerization occurs almost instantaneously. The signal variations show a different behavior for each material system.

The protein solution demonstrates a different behavior, since the change in transmission signal lacks the sharp cutoff. However, a duration without signal variation can be separated from a time regime where the signal is overlaid with clear oscillations (Fig. 4(b)). In this case, VOT was defined as the first local minimum of the oscillating signal. The same oscillations can be observed, when voxels are generated via radical polymerization using Irgacure ${ }^{\circledR}$ OXE01 (Fig. 4(c)). However, in this case no VOT could be assigned, since signal loss occurred from the beginning of the measurement for all applied process parameters. The different results for the different material systems can be explained by their different crosslinking kinetics, whereas the oscillations can be observed as expected for each material system. In the case of radical polymerization, crosslinking occurs in a polymerization chain reaction, which starts if the density of generated radicals reaches a threshold value. This polymerization kinetics can explain the sudden change in signal reduction for the material containing Irgacure $^{\circledR} 184$. It can be expected that the same principle can be applied to the second radical initiator Irgacure ${ }^{\circledR}$ OXE01. And indeed, no slow signal decay was observed. The fact that the signal change occurs almost instantane-

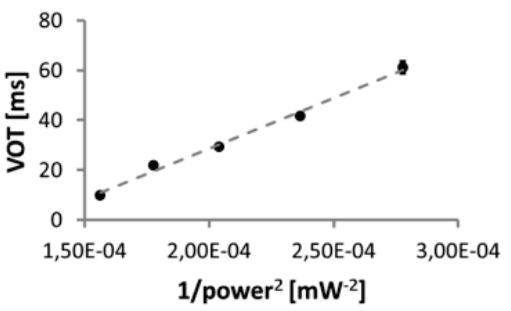

Fig. 5 The VOT is inversely proportional to the squared laser power, as expected for two photon based voxel formation.

ously can be explained by the highly sensitive nature and high dissociation constant that this photoinitiator possesses compared to many other photoinitiators [25]. The kinetics of protein crosslinking follows a different kinetic. Here a step-growth kinetic can be applied which does not have a chain reaction. This different crosslinking kinetics can explain the slowly growing changes in transmission signal.

VOT is strongly linked to the applied laser parameters. Voxel growth is linked to the generation of radicals by the laser irradiation, which depends on a simple rate equation [26]:

$$
\frac{\partial R}{\partial t} \propto \sigma_{2}\left(R_{0}-R\right) \cdot P^{2}
$$

Where $\mathrm{R}$ is the concentration of radicals, $\mathrm{R}_{0}$ the initial concentration of photoinitiator, $\sigma_{2}$ is the effective two photon absorption cross section and $\mathrm{P}$ is the mean laser power. In general, voxel formation occurs, if $\mathrm{R}$ is greater than a material dependent threshold value. Thus, it can be deducted for the VOT by integration:

$$
\operatorname{VOT} \propto\left(\sigma_{2} \cdot P^{2}\right)^{-1}
$$

Therefore, the VOT should be inversely proportional to the squared mean power of the laser beam. This relationship can be confirmed using the protein solution (Fig. 5), which indicates that the VOT is strongly linked to two photon induced voxel formation.

By varying the applied wavelength of the laser source from $690 \mathrm{~nm}$ to $790 \mathrm{~nm}$, the dependency of the VOT on the effective two photon absorption cross section was tested. In this case, the single photon absorption at half the wavelength was taken as an indication for the two photon absorption. The single photon absorption of the photoinitiator possesses a local maximum at approx. $330 \mathrm{~nm}-350 \mathrm{~nm}$ and steadily decays until it reaches a minimum value at approx. $400 \mathrm{~nm}$ (Fig. 6 (a)). VOT measurements were performed with the material containing the same photoinitiator and a strong increase from approx. $10 \mathrm{~ms}$ to $65 \mathrm{~ms}$ could be observed (Fig. 6 (b)). Although, due to the lack of accurate data on the two photon absorption cross section, no quantitative conclusion can be drawn, the qualitative behavior fits the expectations. Moreover, an additional aspect arises when confronted with the measured data. Additionally to the increase in VOT, the standard deviation of the measured values increased significantly. A growing mismatch between applied wavelength and absorption coefficient thus does not only influences the efficiency of voxel formation (longer VOT means longer irradiation time is nec essary for the same result), but also process stability is strongly influenced. This aspect is of importance, if pro- 


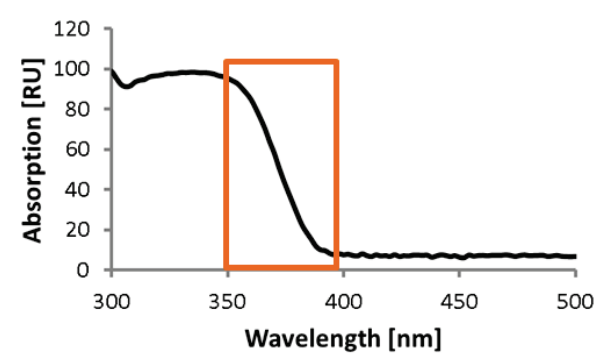

(a)

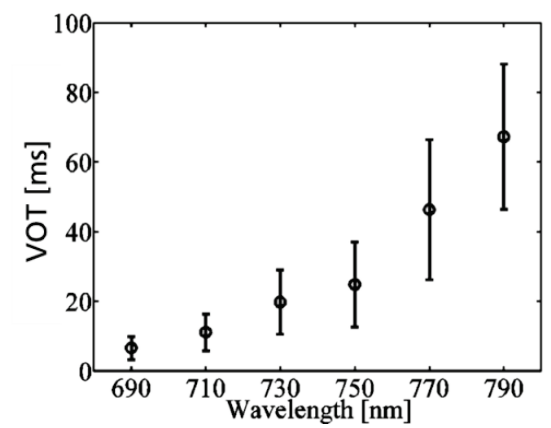

(b)

Fig. 6 (a) Single photon absorption spectrum of the photoinitiator Irgacure ${ }^{\circledR} 184$. The absorption has a local maximum at approx. $330 \mathrm{~nm}-350 \mathrm{~nm}$ and steadily declines until $400 \mathrm{~nm}$. (b) As a consequence the VOT growth steadily, which indicates less efficient voxel formation. Additionally, voxel formation becomes more unstable, since the standard deviation increases drastically.

cessing speed of two photon based lithography should increase in future.

\section{Conclusion and Outlook}

In conclusion, an alternative characterization method for the study of two photon induced voxel formation has been introduced. Based on a simple to implement measurement of the in-process transmitted light, the voxel onset time can be measured. Despite the VOT itself, the recorded signals contain information on the process of voxel formation. A more detailed analysis of these signals may yield the possibility to characterize process features, such as the crosslinking kinetics as shown in this paper.

Due to its possibility to perform measurements during processing, this method may facilitate fast screening of photoinitiators or process parameters to obtain high quality structure in the process of two photon lithography.

\section{Acknowledgments and Appendixes}

This work is funded by the FP7 Projekt ArtiVasc 3D (263416). The above results were acquired using facilities and devices funded by the Federal State of North-Rhine Westphalia and the European Union within the EFRE-program "Regionale Wettbewerbsfähigkeit und Beschäftigung 2007-2013" under grant number 290047022.

\section{References}

[1] Lee, K. S., Kim, R. H., Yang, D. Y., and Park, S. H.: Progress in Polymer Science, 33, (2008) 631.

[2] Maruo, S. and Ikuta, K.: Applied Physics Letters, 76, (2000) 2656.
[3] Ovsianikov, A., Mironov, V., Stampfl, J., and Liska, R.: Expert Review of Medical Devices, 9, (2012) 613.

[4] Sun, H. B. and Kawata, S.: NMR - 3D Analysis - Photopolymerization, 170, (2004) 169.

[5] Ovsianikov, A., Deiwick, A., Van Vlierberghe, S., Pflaum, M., Wilhelmi, M., Dubruel, P., and Chichkov, B.: Materials, 4, (2011) 288.

[6] Seet, K. K., Mizeikis, V., Juodkazis, S., and Misawa, H.: Applied Physics Letters, 88, (2006) 221101.

[7] Sun, H. B., Matsuo, S., and Misawa, H.: Applied Physics Letters, 74, (1999) 786.

[8] von Freymann, G., Ledermann, A., Thiel, M., Staude, I., Essig, S., Busch, K., and Wegener, M.: Advanced Functional Materials, 20, (2010) 1038.

[9] Wylie, R. G., Ahsan, S., Aizawa, Y., Maxwell, K. L., Morshead, C. M., and Shoichet, M. S.: Nature Materials, 10, (2011) 799.

[10] Engelhardt, S., Hoch, E., Borchers, K., Meyer, W., Kruger, H., Tovar, G. E. M., and Gillner, A.: Biofabrication, 3, (2011) 025003.

[11] Engelhardt, S., Hu, Y. L., Seiler, N., Riester, D., Meyer, W., Kruger, H., Wehner, M., Bremus-Kobberling, E., and Gillner, A.: Journal of Laser Micro Nanoengineering, 6, (2011) 54.

[12] Engelhardt, S., Refle, O., and Wehner, M.: Journal of Tissue Engineering and Regenerative Medicine, 6 Suppl. 1, (2012) 299.

[13] Fischer, J. and Wegener, M.: Optical Materials Express, 1, (2011) 614.

[14] Fischer, J. and Wegener, M.: Laser \& Photonics Reviews, (2012) OP65.

[15] Fourkas, J. T.: Journal of Physical Chemistry Letters, $1,(2010) 1221$.

[16] Li, L. J., Gattass, R. R., Gershgoren, E., Hwang, H., and Fourkas, J. T.: Science, 324, (2009) 910.

[17] Formanek, F., Takeyasu, N., Tanaka, T., Chiyoda, K., Ishikawa, A., and Kawata, S.: Optics Express, 14, (2006) 800.

[18] Obata, K., Koch, J., Hinze, U., and Chichkov, B. N.: Optics Express, 18, (2010) 17193.

[19] Ritschdorff, E. T., Nielson, R., and Shear, J. B.: Lab on A Chip, 12, (2012) 867.

[20] Salter, P. S. and Booth, M. J.: Optics Letters, 36, (2011) 2302.

[21] DeVoe R.J., Kalweit H., Leatherdale C.A., and Williams T.R.: Proceedings of SPIE, 4797, (2003) 310.

[22] Sun, H. B., Tanaka, T., and Kawata, S.: Applied Physics Letters, 80, (2002) 3673.

[23] Sun, H. B., Takada, K., Kim, M. S., Lee, K. S., and Kawata, S.: Applied Physics Letters, 83, (2003) 1104.

[24] Sun, H. B., Maeda, M., Takada, K., Chon, J. W. M., Gu, M., and Kawata, S.: Applied Physics Letters, 83, (2003) 819.

[25] Schafer, K. J., Hales, J. M., Balu, M., Belfield, K. D., Van Stryland, E. W., and Hagan, D. J.: Journal of Photochemistry and Photobiology A-Chemistry, 162, (2004) 497.

[26] Serbin, J., Egbert, A.., Ostendorf, A.., Chichkov, B.N., Houbertz,R., Domann,G., Schulz,J., Cronauer,C., Frohlich,L. and Popall,M.: Optics Letters, 28 (2003) 301

(Received: July 23, 2013, Accepted: November 21, 2013) 